\title{
Accretion Disk Oscillations and QPOs in Black Hole X-ray Binaries
}

\author{
Xue-Bing $\mathrm{Wu}$ \\ Department of Astronomy, School of Physics, Peking University, \\ Beijing 100871, China
}

\begin{abstract}
The observed quasi-periodic oscillations (QPOs) in low-mass $\mathrm{X}$-ray binaries have been suggested to be probably related to the characteristic oscillation modes in the inner part of a relativistic accretion disk. Here we study the non-damping oscillation modes in the disk by considering the local perturbations and viscosity effects. We find that the trapped oscillations are amplified significantly when the viscosity parameter increases and the trapped size varies when the radial wavenumber and Mach number change. Our results support that the hundred-hertz QPOs and their low fractional rms amplitudes found in some black hole $\mathrm{X}$-ray binaries may be explained by the trapped non-damping oscillation modes in the inner part of an accretion disk around a rapidly rotating black hole.
\end{abstract}

\section{Introduction}

In recent years, hundred-hertz QPOs have been observed in a few black hole X-ray binaries especially with $R X T E$ satellite. These high-frequency QPOs are probably similar to the kilohertz QPOs observed in many neutron star lowmass X-ray binaries (van der klis 2000). However, the high frequency QPOs in two Galactic microquasars, GRO J1655-40 and GRS 1915+105, are very stable in frequencies at $300 \mathrm{~Hz}, 450 \mathrm{~Hz}$ and $67 \mathrm{~Hz}, 40 \mathrm{~Hz}$ respectively (Morgan, Remillard \& Greiner 1997; Remillard et al. 1999; Strohmayer 2001a,b). These stable QPOs have also very low fractional $\mathrm{rms}$ amplitude $(\sim 1 \%)$. Theoretical models involving frame-dragging (Cui, Zhang \& Chen 1998), diskoseismic gmode (Nowak et al. 1997), and periastro precession (Stella, Vietri \& Morsink 1999) have been proposed to explain these stable QPOs. But the origin of these QPOs is still in debate.

Recently Psaltis \& Norman (2000; hearafter PN) suggested that the observed QPOs in X-ray binaries may be related to the strong resonances peaked at some characteristic oscillation frequencies in the inner part of accretion disks. However, they introduced a simplified term for angular momentum transfer and did not discuss the damping and growth rates of oscillation modes in the disk. It is also important to check if these oscillation modes are damping modes and if some of these modes can be trapped in the inner disk It is also interesting to investigate if the low fractional rms amplitude of QPOs observed in two microquasars can be explained by the trapped feature of some oscillation modes. 


\section{Non-damping Oscillation Modes in Accretion Disk}

The basic equations of a geometrically thin accretion disk are the same as those in PN except that the viscosity term in the angular momentum equation is now expressed as: $N_{\phi}=(1 / \rho r) \partial\left(\nu \rho r^{3} d \Omega / d r\right) / \partial r$. The viscosity is assumed to be described by the usual $\alpha$ prescription, namely, $\nu=\alpha c_{\mathrm{s}} H=\alpha c_{\mathrm{s}}^{2} / \Omega$. Like in $\mathrm{PN}$, we consider the general relativistic effects in an approach to specify the the gravitational potential $\psi$ through the dependences of angular velocity $(\Omega)$, radial and vertical epicyclic frequencies $\left(\kappa\right.$ and $\Omega_{\perp}$ ) on the mass, radius and the spin parameter of the central object. We consider small perturbations to all physical quantities in the form of $Q=Q_{0}+\delta Q e^{i(\omega t-k r-m \phi)}$, where $k$ and $m$ are radial and azimuthal wavenumbers. We also assume that the perturbations do not alter the local sound speed and the disk is isothermal in the vertical direction. Under the local approximation $k r>>1$, we can obtain four perturbed equations and derive the dispersion relation as

$$
\begin{gathered}
\bar{\sigma}^{4}+\alpha \epsilon^{2} \bar{\sigma}^{3}+\left(\bar{\kappa}^{2}+\epsilon^{2}+n \bar{\Omega}_{\perp}^{2}\right) \bar{\sigma}^{2}-\epsilon\left[m \frac{H}{r}\left(\frac{\bar{\kappa}^{2}}{2}-2\right)+2 \alpha \epsilon\left(\frac{\bar{\kappa}^{2}}{2}-2\right)\right. \\
\left.-\alpha \epsilon^{3}-n \epsilon \bar{\Omega}_{\perp}^{2}\right] \bar{\sigma}+n \bar{\kappa}^{2} \bar{\Omega}_{\perp}^{2}=0 .
\end{gathered}
$$

Here we have defined $\bar{\sigma}=i\left(\omega-k u_{r}-m \Omega\right) / \Omega, \bar{\kappa}=\kappa / \Omega, \bar{\Omega}_{\perp}=\Omega_{\perp} / \Omega$, and $\epsilon=k H$. The value of $n$ represents the number of nodes of the oscillations in the vertical direction.

By solving the dispersion relation (1), we found that the characteristic frequencies of oscillation modes depend on the radial wavenumber and radius significantly but are nearly independent of the viscosity. However, the growth (or damping) rates of these modes depend strongly on the viscosity. According to their non-damping feature, we found that the most possible oscillation modes in the disk are the acoustic modes with approximate frequencies of $k u_{r} \pm \sqrt{\kappa^{2}+k^{2} c_{\mathrm{s}}^{2}}$ (in the case of $m=0$ and $n=0$ ) and $k u_{r}+\Omega \pm \sqrt{\kappa^{2}+k^{2} c_{\mathrm{s}}^{2}}$ (in the case of $m=1$ and $n=0$ ), neutral modes with frequency of $k u_{r}$ (in the case of $m=0$ and $n=0$ ) and $k u_{r}+\Omega$ (in the case of $m=1$ and $n=0$ ), and the corrugation modes with approximate frequencies of $k u_{r}+\Omega \pm \Omega_{\perp}$ (in the case of $m=1$ and $n=1$ ). In Figure 1 we show the dependences of oscillation frequencies of all non-damping modes on the disk radius and black hole spin parameters. Note that the lower-frequency acoustic modes and the lower-frequency corrugation mode are trapped in the inner part of accretion disks since $\omega^{2}>\kappa^{2}$. The trapped sizes of these modes depend significantly on the radial wavenumber, Mach number and black hole spin parameter. The relative luminosity variations caused by the oscillations in the trapped area may be estimated using the expression of local flux emitted from a relativistic accretion disk. These relative variations may be comparable to the observed fractional rms magnitudes if the $\mathrm{X}$-ray luminosity variations are caused by the disk oscillations in the trapped area. In Figure 2 we show the trapped sizes of the oscillation modes and the relative luminosity variations. 


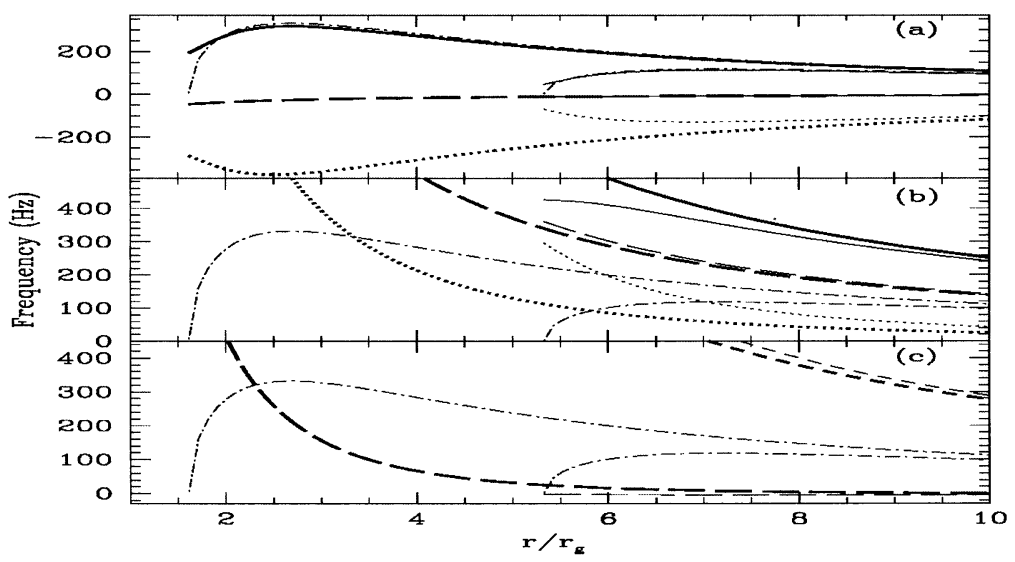

Figure 1. Oscillation frequencies of non-damping modes when $k H=$ 0.1 and $\left|u_{r}\right| / c_{s}=0.3$. The weighted and light lines represent the cases with black hole spin parameter $a_{*}=0.98$ and 0.2 . The panels (a), (b) and $(\mathrm{c})$ correspond to $(\mathrm{m}, \mathrm{n})=(0,0),(1,0)$ and $(1,1)$. In (a) and (b) the solid, dotted and dashed lines represent two acoustic modes and the neutral mode. In (c) the long and short dashed lines represent two corrugation modes. The dot-dashed lines show the radial epicyclic frequencies. The black hole mass was taken to be $7 M_{\odot}$ and $r_{g}$ was defined as $G M / c^{2}$.
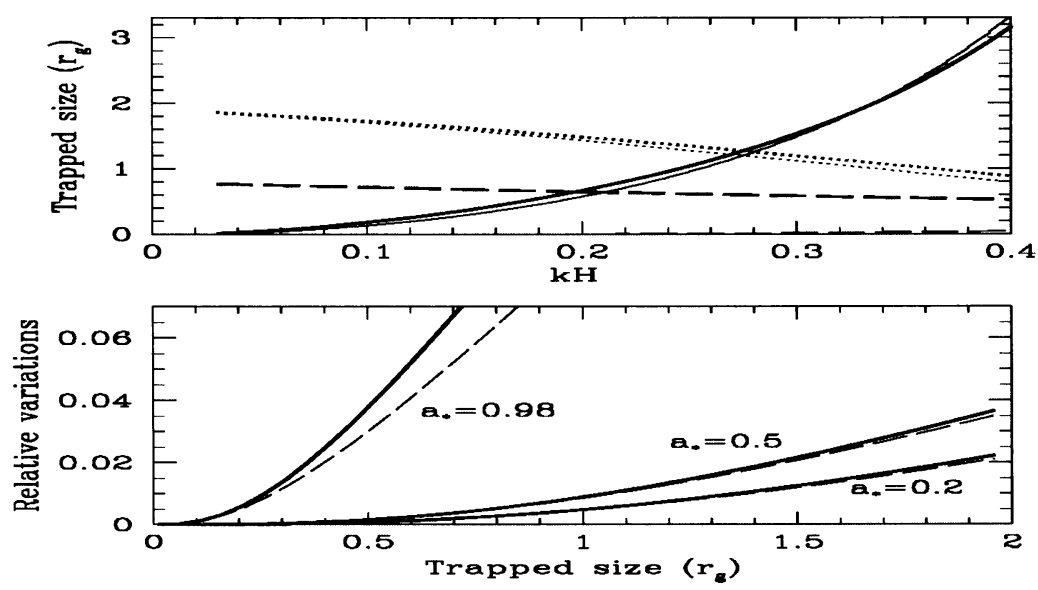

Figure 2. Upper panel: Trapped sizes of three oscillation modes. The solid, dotted and dashed lines represent the lower-frequency acoustic modes of $(\mathrm{m}, \mathrm{n})=(0,0)$ and $(1,0)$, and the lower-frequency corrugation mode of $(\mathrm{m}, \mathrm{n})=(1,1)$. The weighted and light lines correspond $a_{*}=$ 0.98 and 0.2 . Lower panel: Relative variations caused by the trapped oscillations. The solid and dashed lines correspond to the cases of accretion rate of $10^{17} \mathrm{~g} / \mathrm{s}$ and $10^{18} \mathrm{~g} / \mathrm{s}$. 


\section{Conclusion}

RXTE observations on GRO J1655-40 have shown $300 \mathrm{~Hz}$ and $450 \mathrm{~Hz}$ QPOs with very low $(\sim 1 \%)$ rms magnitude. If these stable QPOs originate from the trapped disk oscillations, from Figures 1 and 2 we see that it may be explained by either the lower-frequency acoustic mode in the case of $m=1$ and $n=0$ or the lowerfrequency corrugation mode in the case of $m=n=1$ and $a_{*} \sim 0.9$. Considering the co-existence of lower frequency QPOs $(\tilde{9} \mathrm{~Hz})$, the rapidly spinning black hole is more favored to explain the high QPO frequency. Similar conclusion may be obtained for other black hole X-ray binaries, especially GRS 1915+105. However, global investigations on the oscillation feature of the disk modes and the unambiguous determinations of the black hole spin are still needed to confirm this explanation. Further studies on the origin of low-frequency QPOs and their correlation with high-frequency QPOs, as well as the correlation between spectral and temporal properties, will help us to understand more clearly the origin of stable QPOs in black hole X-ray binaries.

We note that the high-frequency QPOs observed in other two black hole X-ray binaries, XTE J1550-564 and XTE J1859+226, are variable in frequency from 102 to $284 \mathrm{~Hz}$ and from 82 to $187 \mathrm{~Hz}$ respectively (Remillard et al. 1999; Homan et al. 2000; Cui et al. 2000). This seems to be in contrast to the stable hundred hertz QPOs observed in GRO J1655-40 and GRS 1915+50. If we try to explain the variable QPO frequency of XTE J1550-564 and XTE J1859+226 according to the trapped modes in the inner disc, we need to assume larger trapping size $\left(>r_{g}\right)$ of oscillation modes. This is still possible if the Mach number of accretion flow is lower and the spin parameter of black hole is larger. The variable QPO frequency may be explained by the moving of the inner disc radius within the trapped area. Further detailed analyses of X-ray spectra using more realistic disc model are needed to confirm this conclusion.

\section{References}

Cui, W., Shrader, C.R., Haswell, C.A., Hynes, R., 2000, ApJ, 535, L123

Cui, W., Zhang, S.N., \& Chen, W. 1998, ApJ, 492, L53

Homan, J., Wijnands, R., van der Klis, M., Belloni, T., van Paradijs, J., KleinWolt, M., Fender, R., Méndez, M., ApJS, 132, 377

Morgan, E.H., Remillard, R.A., \& Greiner, J. 1997, ApJ, 482, 993

Nowak, M.A., et al. 1997, ApJ, 477, L91

Psaltis, D., \& Norman, C. 2001, ApJ, accepted

Remillard, R.A., et al. 1999, ApJ, 522, 397

Stella, L., Vietri, M., \& Morskink, S.M. 1999, ApJ, 524, L63

Strohmayer, T.E. 2001a, ApJ, 552, L49

Strohmayer, T.E. 2001b, ApJ, 554, L169

van der Klis, M., 2000, ARA\&A, 38, 71 